\title{
X-Ray Determination of the Disorder in Poly(oxymethylene) Crystals
}

\author{
Yuji Aoki, Akio Chiba, and Motozo Kaneko \\ Department of Polymer Science, Faculty of Science, \\ Hokkaido University, Sapporo, Japan.
}

(Received June 17, 1970)

\begin{abstract}
S: Ruland's concept of the isotropic disorder function is used to estimate the disorder parameter and degree of crystallinity in $\gamma$-ray irradiated and solution-grown poly(oxymethylene)s. The degree of crystallinity increases with increasing irradiation dosage, but the disorder parameter remains constant. The disorder is attributed to thermal motions, the root mean squared amplitude being about $0.2 \AA$ at room temperature. The increase in crystallinity is mainly caused by degraded molecular chains in the disordered region. The disorder parameter of the solution-grown crystal is larger than that of $\gamma$-ray irradiated samples and decreases with annealing. Increase in the disorder parameter in the solution-grown crystal may be caused by the lattice imperfections in the crystal. In both samples the degree of crystallinity decreases above $70^{\circ} \mathrm{C}$, and at $120^{\circ} \mathrm{C}$ it is $5 \%$ less than the value at room temperature. In this temperature range, partial melting at the surface layer of the crystal may take place.

KEY WORDS X-Ray / Crystallinity / Lattice Disorder / Thermal

Motion / Irradiation / Solution-Grown Crystal / Annealing / Partial

Melting / Poly(oxymethylene) /
\end{abstract}

Ruland $^{1,2}$ has developed an X-ray method of crystallinity determination which takes into account the diffuse scattering due to thermal motions and lattice imperfections in a crystal. Using his method, the degree of crystallinity and the disorder parameter in polyethylene, ${ }^{1,3,4}$ polypropylene, ${ }^{1}$ and other crystalline polymers ${ }^{2,5,6}$ have been measured. It has been found that the disorder parameter is predominantly determined by the thermal motions in linear polymers in which the interchain attraction is due to van der Waals force only. ${ }^{1}$

Recently, Davis, et al. ${ }^{7}$ reported that a unit cell dimension of polyethylene is not unique but varies systematically with crystallization conditions, annealing and deformation. It is interesting to investigate whether the disorder parameter also varies with such treatment.

In the present study, $\gamma$-ray irradiated and solution-grown poly(oxymethylene)s (POM) were used. It would be useful to measure the disorder parameter of the solution-grown crystal, since the disorder parameter has not previously been discussed in respect of samples crystallized from solution.
The influences of crystallization conditions and the effect of $\gamma$-ray irradiation on the disorder in a crystal and on the degree of crystallinity are investigated. The disorder parameter and degree of crystallinity vary with the $\gamma$-ray irradiation, crystallization conditions and the annealing. The temperature dependence on the disorder parameter and on the degree of crystallinity is also shown. The mechanism of those variations is discussed in terms of thermal motions and lattice imperfections.

\section{THEORY}

According to Ruland, ${ }^{1}$ the degree of crystallinity $x_{c r}$ is given by the expression

$$
x_{c r}=K \frac{\int_{0}^{\infty} s^{2} I_{c r}(s) \mathrm{d} s}{\int_{0}^{\infty} s^{2} I(s) \mathrm{d} s}
$$

where $s$ is the reciprocal space vector $(s=2 \sin \theta / \lambda)$, $I_{c r}(s)$ and $I(s)$ intensities of the coherent scattering at $s$ in the crystalline and total regions, respectively. $K$ is a constant of proportionality 
to correct the crystallinity for the disorder function and is given by

$$
K=\frac{\int_{0}^{\infty} s^{2} \overline{f^{2}} \mathrm{~d} s}{\int_{0}^{\infty} s^{2} \overline{f^{2} D} \mathrm{~d} s}
$$

where $\overline{f^{2}}$ is the mean squared amplitude of atomic scattering $\left(\bar{f}^{2}=\sum_{i} N_{i} f_{i}^{2} / \sum_{i} N_{i}\right), N_{i}$ being the number of atoms of type $i$ with a scattering factor $f_{i} . \quad D$ is the disorder function that represents the overall disorder in the crystalline part of a substance. For lattice imperfections of the first kind (in which the long-range order is conserved), including thermal motions, $D$ can be approximated well with a Gaussian curve. However for imperfections of the second kind (in which the longrange order is destroyed, "paracrystal") the theory shows that $D$ conforms more closely to the expression ${ }^{8}$

$$
D=\frac{2 \exp \left(-a s^{2}\right)}{1+\exp \left(-a s^{2}\right)}
$$

To solve eq 1 , the disorder function $D$ has to be determined and this is done as follows. When integration intervals over larger regions of $s$ are chosen, the next equation is satisfied independent of the crystallinity of the substance.

$$
\int_{s_{0}}^{s_{p}} s^{2} I(s) \mathrm{d} s=\int_{s_{0}}^{s_{p}} s^{2} \overline{f^{2}} \mathrm{ds}
$$

These regions can be found experimentally by studying samples with different crystallinities. The upper limits $s_{p}$ can be varied. Thus the degree of crystallinity is constant for a series of such integration intervals.

$$
\begin{gathered}
x_{c r}=K\left(s_{0}, s_{p}, D, \overline{f^{2}}\right) \frac{\int_{s_{0}}^{s_{p}} s^{2} I_{c r}(s) \mathrm{d} s}{\int_{s_{0}}^{s_{p}} s^{2} I(s) \mathrm{d} s}=\mathrm{constant} . \\
K\left(s_{0}, s_{p}, D, \overline{f^{2}}\right)=\frac{\int_{s_{0}}^{s_{p}} s^{2} \overline{f^{2}} \mathrm{~d} s}{\int_{s_{0}}^{s_{p}} s^{2} \overline{f^{2}} D \mathrm{~d} s} \\
\int_{c r}^{\prime}=\frac{\int_{s_{0}}^{s_{p}} s^{2} I_{c r}(s) \mathrm{d} s}{\int_{s_{0}}^{s_{p}} s^{2} I(s) \mathrm{d} s}
\end{gathered}
$$

Having found a series of such regions, we can determine the series of $K$ values which yields $x_{c r}=$ constant for a series of $D$-function.

Ruland $^{2}$ found that calculated values of the coefficient

$$
K=\frac{\int_{s_{0}}^{s_{p}} s^{2} \overline{f^{2}} \mathrm{~d} s}{\int_{s_{0}}^{s_{p}} s^{2} \overline{f^{2}} D \mathrm{~d} s}
$$

when plotted as a function of $s_{p}$, the upper limit of integration, gave approximately straight lines with the same slope regardless of whether $D$ was assumed to be given by eq 3 or by a Gaussian function. This showed that all kinds of displacements of the atoms from their ideal positions can be lumped together in one Gaussian lattice-imperfection factor

$$
D=\exp \left(-k s^{2}\right)
$$

in which $k$ is the sum of three terms due to thermal motions $\left(k_{\mathrm{T}}\right)$ and to lattice imperfections of the first $\left(k_{\mathrm{I}}\right)$ and second $\left(k_{\mathrm{II}}\right)$ kinds.

$$
k=k_{\mathrm{T}}+k_{\mathrm{I}}+k_{\mathrm{II}}
$$

\section{MATERIALS}

The samples used in this work are two series of POM. One series comprises $\gamma$-ray irradiated samples designated A-1, A-2, and A-3. The other is solution-grown samples named B-1, B-2, B-2', and B-2 $2^{\prime \prime}$. Details of these samples are summarized in Table $\mathrm{I}$.

Table I.

Sample A-1 A commercial powder POM (Delrin, M.W. 29000) annealed 10 hours at $130^{\circ} \mathrm{C}$

A-2 The same, $4.1 \times 10^{5}-\mathrm{R}$ irradiation

A-3 The same, $9.8 \times 10^{6}-\mathrm{R}$ irradiation

Sample B-1 Solution-grown POM (crystallized from $0.03-\%$ cyclohexanol solution at $100^{\circ} \mathrm{C}$ )

B-2 Solution-grown POM (crystallized from $0.03-\%$ cyclohexanol solution cooled slowly from $100^{\circ} \mathrm{C}$ )

B-2' The same, annealed 10 hours at $130^{\circ} \mathrm{C}$

B-2" The same, annealed 20 hours at $140^{\circ} \mathrm{C}$ 
Solution-grown crystals were prepared from a 0.03-wt\% cyclohexanol solution of $\mathrm{du}$ Pont Delrin 150X. Sample B-1 was separated from the solvent by gradual cooling. Sample B-2 was isothermally separated at a temperature of $100^{\circ} \mathrm{C}$. The suspension of the crystals was slowly filtered through the glass filter. The solution-grown crystals were dried by evacuation in a desiccator for one week at about $60^{\circ} \mathrm{C}$. The solvent content in the crystals was found to be negligibly small by infrared measurements.

\section{EXPERIMENTAL PROCEDURE}

$X$-ray measurements were performed with a Rigaku Denki diffractometer using a scintillation counter with a pulse-height analyzer and copper radiation. The monochromatization was carried out with the diffracted-beam monochromatization technique. ${ }^{9}$ The radiation scattered was measured with a point-to-point technique and a fixed-time method which ensured close accuracy in intensity estimation and an easy conversion of the diffractogram obtained in Bragg angle into a reciprocal space vector chart. Intensity was corrected only for polarization.

The arrangement of the diffracted-beam monochromatization technique is shown schematically in Figure 1. The LiF crystal was so cut and bent that $\mathrm{CuK}_{\alpha}$ radiation was brought to a focus in the plane of the receiving slit with $0.6-\mathrm{mm}$ thickness. Compton scattering from the sample was practically eliminated by the receiving slit

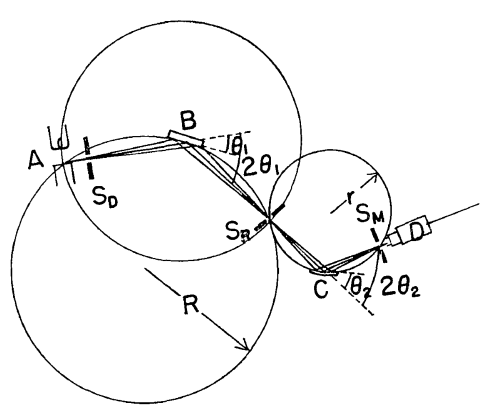

Figuure 1. Schematic diagram of the apparatus: A, X-ray tube; B, sample; C, LiF curved crystal; $\mathrm{D}$, scintillation counter; $\theta_{1}$, rotation angle of sample; $\theta_{2}$, rotation angle of $\mathrm{LiF}$ crystal; $\mathbf{R}$, radius of first focal circle $(=185 \mathrm{~mm}$ ); $\mathrm{r}$, radius of second focal circle $(=50 \mathrm{~mm}) ; \mathrm{S}_{D}$, goniometer divergence-slit; $\mathrm{S}_{M}$, monochrometer receiver-slit. in the region where Compton scattering was appreciable, even if it were reflected from the mosaic structure of the LiF crystal. This technique is, therefore, appropriate for Ruland's method.

The separation lines between the crystalline peaks and continuous scattering have been established by drawing smooth curves from tail to tail following the general slope of the continuous scattering. Thus it should be emphasized that the degree of crystallinity, as measured in this study, is still probably a minimum value.

The sample was heated by a heater mounted behind the sample holder and the temperature of the sample was measured with a copperconstantan thermocouple.

\section{EXPERIMENTAL RESULTS}

\section{Determination of the Disorder Parameter}

Selection of independent regions of crystallinity for use in eq 5 was required. $s_{0}$ was taken as 0.1 (as was done by Ruland) and for $s_{p}$ were chosen three values such as $0.4,0.8$, and 1.1 . These could be readily found by empirical means, in the case of the series of POM samples, using a planimeter and the $s^{2} I(s)$ plot. Comparison of the integrals in $s^{2} I(s)$ with those in $s^{2} \overline{f^{2}}$ is shown in Table II. The integrated in-

Table II. Integrated intensities at each interval for A-series samples at varying temperatures

\begin{tabular}{cccc}
\hline$s_{0}-s_{p}$ & $0.1-0.4$ & $0.1-0.8$ & $0.1-1.1$ \\
\hline $\mathrm{A}-1\left(18^{\circ} \mathrm{C}\right)$ & 243 & 533 & 754 \\
$\left(110^{\circ} \mathrm{C}\right)$ & 249 & 536 & 754 \\
$\mathrm{~A}-2\left(18^{\circ} \mathrm{C}\right)$ & 245 & 538 & 754 \\
$\left(110^{\circ} \mathrm{C}\right)$ & 257 & 558 & 754 \\
$\mathrm{~A}-3\left(18^{\circ} \mathrm{C}\right)$ & 243 & 539 & 754 \\
$\left(\int_{s_{0}}^{s_{p}} s^{2} \overline{f^{2}} \mathrm{~d} s\right)$ & 137 & 486 & 754 \\
\hline
\end{tabular}

tensities $\int_{s_{0}}^{s_{p}} s^{2} I(s) \mathrm{d} s$ in these regions were kept constant independent of the degree of crystallinity. For lower values of the upper limit $s_{p}$, $\int_{s_{0}}^{s_{p}} s^{2} \overline{f^{2}} \mathrm{~d} s$ values are about $40 \%$ smaller than $\int_{s_{0}}^{s_{p}} s^{2} I(s) \mathrm{d} s$, since the intensity of the 
X-Ray Determination of the Disorder in Polyoxymethylene Crystals

Table III. Degree of crystallinity as a function of $k$ and integration interval for Sample A-1

\begin{tabular}{ccccccc}
\hline$s_{0}-s_{p}$ & $k=0$ & $k=1.0$ & $k=1.5$ & $k=1.7$ & $k=2.0$ & $k=2.5$ \\
\hline $0.1-0.4$ & 64.5 & 70.2 & 73.5 & 74.8 & 77.0 & 80.4 \\
$0.1-0.8$ & 45.2 & 60.1 & 68.6 & 72.2 & 77.9 & 87.6 \\
$0.1-1.1$ & 36.2 & 57.4 & 69.9 & 75.1 & 83.4 & 97.0 \\
& & & Mean $x_{c r} 74 \%$ & & \\
\hline
\end{tabular}

Bragg reflection is very strong. However, these discrepancies cannot affect the results very much since in both fractions the numerator and denominator behave similarly as functions of the upper limit of the integration and also because the deviation of $\overline{f^{2}}$ from its experimental values is restricted to small values of $s$ where the $D$ function is close to unity.

The results of the application of eq 5 , with the $D$-function taken as $\exp \left(-k s^{2}\right)$ for sample A-1 at room temperature, are shown in Table III.

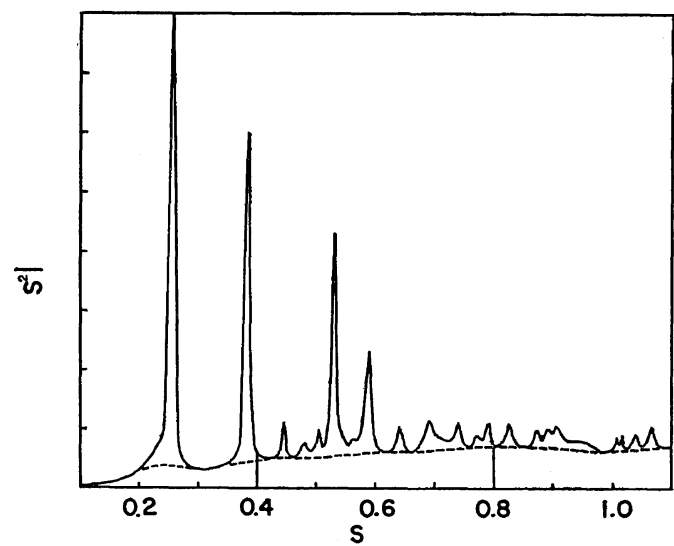

Figure 2. $s^{2} I(s)$ vs. $s$ plot of polyoxymethylene (sample A-2). Dotted line denotes the separation line between the crystalline peaks and the continuous scattering.
It can be seen that by taking $k=1.7$, fairly constant crystallinity is obtained from different regions of integration. There is a tendency towards smaller values in the medium region. But since the deviations in $x_{c r}$ are not significant, an isotropic $D$-function can be regarded as a good approximation.

$\gamma$-Ray Irradiation POM

Table IV shows the disorder parameter and the degree of crystallinity in A-series POM at room temperature. The disorder parameter is constant independent of the irradiation dosage. On the other hand, the degree of crystallinity increases with increasing irradiation: $74 \%$ for the original sample and $80 \%$ for the $9.8 \times 10^{6}-\mathrm{R}$ irradiated sample.

The temperature dependence on the disorder parameter and on the degree of crystallinity are shown in Figure 3. The black circles indicate the original sample (A-1) and the white circles the $4.1 \times 10^{5}-\mathrm{R}$ irradiated samle (A-2). Both A-1 and A-2 show similar tendencies. With rising temperature, the disorder parameter increases linearly up to about $100^{\circ} \mathrm{C}$ and abruptly increases above $100^{\circ} \mathrm{C}$, while the degree of crystallinity remains constant in the neighborhood of $70^{\circ} \mathrm{C}$ and decreases gradually with higher temperatures. The degree of crystallinity at $120^{\circ} \mathrm{C}$ is $5 \%$ lower than that at room temperature.

Table IV. Crystallinity as function of $k$ and integration interval for $\gamma$-ray irradiated poly(oxymethylene)s

\begin{tabular}{|c|c|c|c|c|c|c|}
\hline \multirow{2}{*}{$\begin{array}{c}\text { Interval } \\
s_{0}-s_{p}\end{array}$} & \multicolumn{2}{|c|}{ Sample A-1 } & \multicolumn{2}{|c|}{ Sample A-2 } & \multicolumn{2}{|c|}{ Sample A-3 } \\
\hline & $k=0$ & $k=1.7$ & $k=0$ & $k=1.7$ & $k=0$ & $k=1.7$ \\
\hline $0.1-0.4$ & 64.5 & 74.8 & 66.9 & 77.6 & 69.6 & 80.7 \\
\hline $0.1-0.8$ & 45.5 & 72.2 & 47.2 & 75.0 & 48.0 & 76.3 \\
\hline $0.1-1.1$ & 36.2 & 75.1 & 37.3 & 77.4 & 38.4 & 79.7 \\
\hline Mean $x_{c r}$ & & $74 \%$ & & $77 \%$ & & $80 \%$ \\
\hline
\end{tabular}




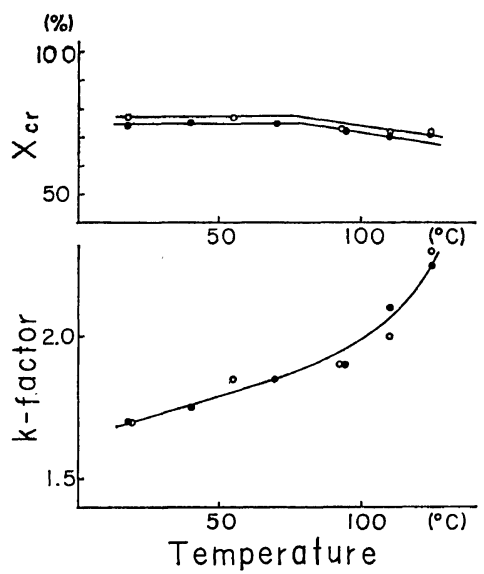

Figure 3. The temperature dependence of the degree of crystallinity and the disorder parameter for samples A-1 and A-2: $0, \mathbf{A}-1 ; \bigcirc, \mathbf{A}-2$.

\section{Solution-Grown POM}

The disorder parameter and the degree of crystallinity for solution-grown crystals and their annealed samples are summarized in Table V. In the sample B-1 crystallized at the constant temperature $100^{\circ} \mathrm{C}, k$ is 1.9 and $x_{c r}$ is $76 \%$ and in sample B-2, precipitated over gradual cooling from $100^{\circ} \mathrm{C}, k$ is 2.1 and $x_{c r}$ is $72 \%$. A sample crystallized at a constant temperature has a higher degree of crystallinity and a lesser degree of disorder. Upon annealing, the disorder parameter decreases and the degree of crystallinity increases. However, the disorder parameter of the B-series is larger than that of the A-series samples.

The temperature dependence on the degree of crystallinity and on the disorder parameter for sample B-1 are shown in Figure 4. The degree of crystallinity decreases at $100^{\circ} \mathrm{C}$. This behavior is the same as that of A-series samples, whereas the disorder parameter does not show

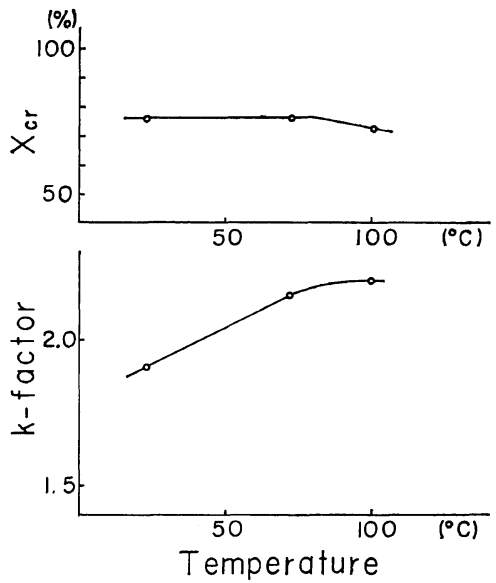

Figure 4. The temperature dependence of the degree of crystallinity and the disorder parameter for the sample B-1.

the sharply increasing values above $100^{\circ} \mathrm{C}$ as occur in the A-series.

\section{DISCUSSION}

The disorder parameters in the A-series samples are exactly the same $(k=1.7)$ as each other at room temperature, despite their varying irradiation treatment. This value indicates that the disorder in the POM crystal is due to thermal motions and the effect of the lattice imperfections is small compared with that of thermal motions. The reason is as follows. If only thermal motions are involved in the disorder parameter, $k$ is identical with a half of an isotropic Debye-Waller factor $B=8 \pi^{2}\left\langle\Delta u^{2}\right\rangle$, where $\left\langle\Delta u^{2}\right\rangle$ is the mean square amplitude of thermal motions. With the $k$ value of 1.7 , the root mean square amplitude is calculated at about $0.2 \AA$. It agrees with the value which was

Table V. Crystallinity as function of $k$ and integration interval for solution-grown poly(oxymethylene)s

\begin{tabular}{|c|c|c|c|c|c|c|c|c|}
\hline \multirow{2}{*}{$\begin{array}{c}\text { Interval } \\
s_{0}-s_{p}\end{array}$} & \multicolumn{2}{|c|}{ Sample B-1 } & \multicolumn{2}{|c|}{ Sample B-2 } & \multicolumn{2}{|c|}{ Sample B-2' } & \multicolumn{2}{|c|}{ Sample B-2" } \\
\hline & $k=0$ & $k=1.9$ & $k=0$ & $k=2.1$ & $k=0$ & $k=1.9$ & $k=0$ & $k=1.85$ \\
\hline $0.1-0.4$ & 65.6 & 77.6 & 60.4 & 72.7 & 64.0 & 75.7 & 64.2 & 75.6 \\
\hline $0.1-0.8$ & 43.7 & 72.9 & 39.5 & 69.5 & 43.3 & 72.3 & 44.2 & 72.9 \\
\hline $0.1-1.1$ & 34.8 & 77.2 & 30.7 & 72.8 & 33.8 & 75.0 & 34.5 & 75.3 \\
\hline Mean $x_{c r}$ & & $76 \%$ & & $72 \%$ & & $74 \%$ & & $75 \%$ \\
\hline
\end{tabular}


evaluated from the temperature dependence of the intensities of several Bragg reflections. ${ }^{10}$ On the crystal structure analysis of POM, Carazzolo ${ }^{11}$ found an isotropic temperature factor with a $B$-value of $2.3 \AA^{2}$. Uchida and Tadokoro $^{12}$ carried out the crystal structure analysis of POM obtained by solid state polymerization of single crystals of tetraoxane. The averaged value of the anisotropic temperature factor is about $4 \AA^{2}$. These values are in close agreement with our value of $3.4 \AA^{2}$.

The chemical and physical changes occuring in the processes of $\gamma$-ray irradiation are rather complicated. It has been reported that the solution viscosity of POM decreases remarkably with irradiation. ${ }^{13}$ It was shown that the $\gamma$-ray irradiation degrades the covalent bond of main chains. Miki, et al. ${ }^{14}$ have found from dynamic mechanical measurements that the real part of complex rigidity decreases with increasing irradiation dosages. They expected lattice defects to have been produced by irradiation in the crystalline region. Our results indicate that the degree of crystallinity increases with irradiation. It shows that POM chains mainly degrade in the disordered regions rather than in crystalline regions, since the degradation in the crystalline region must lower the crystallinity. ${ }^{15}$ The chain scission reactions induce the chain rearrangement and/or the vaporization of degraded molecules. In fact, it was found that the gases were produced with $9.8 \times 10^{6}-\mathrm{R}$ irradiation. Chain scissions of folded chains and of tie molecules near the surface layer of lamellae may be expected. Nevertheless, the disorder parameter remains constant. It is therefore suggested that the structure change in these areas does not affect the disorder parameter or thermal motions in the crystalline region.

Nuclear magnetic resonance studies ${ }^{16}$ have been carried out on the A-series of POM investigated in this work. It has been found that the mobile fraction $\left(f_{m}\right)$ is $0.26,0.23$, and 0.15 at $20^{\circ} \mathrm{C}$ for Samples A-1, A-2, and A-3, respectively. Considering that the degree of crystallinity corresponds to $\left(1-f_{m}\right)$, these values are in close agreement with the degrees of crystallinity obtained from the X-ray measurements.

The disorder parameter of solution-grown crystals has a higher value than that of the
A-series samples. This indicates that solutiongrown crystals include lattice imperfections. For the same crystallized by gradual cooling (B-2), more lattice imperfections are included than for the sample crystallized at constant temperature. Upon annealing at higher temperature, the disorder parameter decreases as shown in Table V. These facts suggest that the solution-grown crystals precipitated at lower temperature are less stable but that their stability increases with annealing. Such a conclusion is supported by the fact that the unit cell dimension of solution-grown polyethylene varies considerably with annealing without any change in crystal thickness but that of melt-grown polyethylene does not alter with annealing if the crystal thickness does not increase. ${ }^{7}$

The degree of crystallinity of solution-grown crystals ranges from 72 to $76 \%$. These values seem small, although the degree of crystallinity measured in this study is probably a minimum value. However, the location of the amorphous region is not yet clearly understood.

The degree of crystallinity in both the A-series and the B-series samples decreases above $70^{\circ} \mathrm{C}$. The decrease in crystallinity may be considered to have resulted from the partial melting of the POM crystal. This is justified from the temperature dependence of the intensity of X-ray small angle scattering. Nobuta, et al. ${ }^{17}$ in our laboratory found that the variation in the intensity could not be explained by the electron density difference between the crystalline and the amorphous regions at higher temperatures, and suggested that partial melting took place in this temperature range. The disorder parameter increases sharply with partial melting. It is reasonable to expect that the disorder parameter in the crystals increases with increasing partial melting. A detailed study of the partial melting of POM will be presented in the near future.

In conclusion, the disorder parameter in the A-series samples is due mainly to thermal motions whose root mean squared amplitude is about $0.2 \AA$ at room temperature, and which are not affected by $\gamma$-ray irradiation. Secondly, solution-grown crystals contain more lattice imperfections, but these imperfections are removed by annealing. Thirdly, partial melting in POM crystals occurs from $70^{\circ} \mathrm{C}$. 


\section{REFERENCES}

1. W. ${ }_{-}$Ruland, Acta Cryst., 14, 1180 (1961).

2. W. Ruland, Polymer, 5, 89 (1964).

3. H. G. Kilian, Kolloid-Z. Z. Polym., 183, 1 (1962).

4. W. Glenz, H. G. Kilian, and F. H. Müller, Kolloid-Z. Z. Polym., 206, 104 (1965).

5. A. Viswanathan and V. Venkatakrishnan, $J$. Appl. Polym. Sci., 131, 785 (1969).

6. D. Kockkott, Kolloid-Z. Z. Polym., 214, 31 (1966).

7. G. T. Davis, R. K. Eby, and G. M. Martin, J. Appl. Phys., 39, 4973 (1968).

8. R. Hosemann, Z. Phys., 128, 1, 465 (1950).

9. A. R. Lang, Rev. Sci. Instr., 27, 17 (1956).
10. A. Chiba, Y. Aoki, and M. Kaneko, Rep. Progr. Polym. Phys. Japan, 11, 213 (1968).

11. G. A. Carazzolo, J. Polym. Sci., Part A-1, 1 1573 (1963).

12. T. Uchida and H. Tadokoro, J. Polym. Sci., Part A-2, 5, 63 (1967).

13. S. Torikai, J. Polym. Sci., Part A-2, 2239 (1964).

14. K. Miki, Y. Yokokawa, K. Hikichi, and J. Furuichi, Japan. J. Appl. Phys., 5, 818 (1966).

15. W. Jaffe and B. Wunderlich, J. Polym. Sci., Part A-2, 6, 825 (1968).

16. A. Tsutsumi, private communication.

17. A. Nobuta, A. Chiba, and M. Kaneko, Rep. Progr. Polym. Phys. Japan, 12, 137 (1969). 